\title{
Integrability Formulas. Part II
}

\author{
Bo Li \\ $\mathrm{Na} \mathrm{Ma}$ \\ Qingdao University of Science \\ and Technology \\ China \\ Qingdao University of Science \\ and Technology \\ China \\ Xiquan Liang \\ Qingdao University of Science \\ and Technology \\ China
}

Summary. In this article, we give several differentiation and integrability formulas of special and composite functions including trigonometric function, and polynomial function.

MML identifier: INTEGR13, version: $\underline{7.11 .07 \quad 4.156 .1112}$

The terminology and notation used here have been introduced in the following articles: [12], [13], [2], [3], [9], [1], [6], [11], [14], [4], [18], [7], [8], [5], [19], [10], [16], [17], and [15].

For simplicity, we use the following convention: $a, x$ are real numbers, $n$ is an element of $\mathbb{N}, A$ is a closed-interval subset of $\mathbb{R}, f, h, f_{1}, f_{2}$ are partial functions from $\mathbb{R}$ to $\mathbb{R}$, and $Z$ is an open subset of $\mathbb{R}$.

The following propositions are true:

(1) Suppose that

(i) $A \subseteq Z$,

(ii) $f=\frac{1}{\text { (the function sin) (the function } \cos )}$,

(iii) $Z \subseteq \operatorname{dom}(($ the function $\ln ) \cdot($ the function $\tan ))$,

(iv) $Z=\operatorname{dom} f$, and

(v) $f$ is continuous on $A$.

Then $\int_{A} f(x) d x=(($ the function $\ln ) \cdot($ the function $\tan ))(\sup A)-(($ the function $\ln ) \cdot($ the function $\tan ))(\inf A)$. 
(2) Suppose that

(i) $A \subseteq Z$,

(ii) $f=-\frac{1}{\text { (the function } \sin )(\text { the function } \cos )}$,

(iii) $Z \subseteq \operatorname{dom}(($ the function $\ln ) \cdot($ the function $\cot ))$,

(iv) $Z=\operatorname{dom} f$, and

(v) $f$ is continuous on $A$.

Then $\int_{A} f(x) d x=(($ the function $\ln ) \cdot($ the function $\cot ))(\sup A)-(($ the function $\ln ) \cdot($ the function $\cot ))(\inf A)$.

(3) Suppose that

(i) $A \subseteq Z$,

(ii) $f=2($ (the function $\exp )$ (the function $\sin )$ ),

(iii) $Z \subseteq \operatorname{dom}(($ the function $\exp )$ ((the function $\sin )-($ the function $\cos )))$,

(iv) $Z=\operatorname{dom} f$, and

(v) $f$ is continuous on $A$.

Then $\int_{A} f(x) d x=(($ the function $\exp ) \quad(($ the function sin $)-($ the function $\cos )))(\sup A)-(($ the function $\exp ) \quad(($ the function $\sin )-($ the function $\cos )))(\inf A)$.

(4) Suppose that

(i) $A \subseteq Z$,

(ii) $f=2($ (the function $\exp )$ (the function $\cos )$ ),

(iii) $Z \subseteq \operatorname{dom}(($ the function $\exp )(($ the function $\sin )+($ the function $\cos )))$,

(iv) $Z=\operatorname{dom} f$, and

(v) $f$ is continuous on $A$.

Then $\int_{A} f(x) d x=(($ the function $\exp ) \quad(($ the function $\sin )+($ the function $\cos )))(\sup A)-(($ the function $\exp ) \quad(($ the function $\sin )+($ the function $\cos )))(\inf A)$.

(5) Suppose $A \subseteq Z=\operatorname{dom}($ (the function $\cos )-($ the function $\sin )$ ) and (the function cos)-(the function sin) is continuous on $A$. Then $\int_{A}(($ the function $\cos )-($ the function $\sin ))(x) d x=(($ the function $\sin )+($ the function $\cos ))(\sup A)-(($ the function $\sin )+($ the function $\cos ))(\inf A)$.

(6) Suppose $A \subseteq Z=\operatorname{dom}($ (the function $\cos )+($ the function $\sin )$ ) and (the function cos) $+($ the function $\sin$ ) is continuous on $A$. Then $\int_{A}(($ the function $\cos )+($ the function $\sin ))(x) d x=($ the function $\sin )-($ the function $\cos ))(\sup A)-(($ the function $\sin )-($ the function $\cos ))(\inf A)$. 
(7) Suppose $Z \subseteq \operatorname{dom}\left(\left(-\frac{1}{2}\right) \frac{\text { (the function } \sin )+(\text { the function } \cos )}{\text { the function } \exp }\right)$. Then

(i) $\quad\left(-\frac{1}{2}\right) \frac{\text { (the function } \sin )+(\text { the function } \cos )}{\text { the function } \exp }$ is differentiable on $Z$, and

(ii) for every $x$ such that $x \in Z$ holds $\left(\left(-\frac{1}{2}\right) \frac{\text { (the function sin) }+(\text { the function } \cos )}{\text { the function } \exp }\right)^{\prime}{ }_{\mid Z}(x)=\frac{\text { (the function } \sin )(x)}{\text { (the function } \exp )(x)}$.

(8) Suppose that

(i) $A \subseteq Z$,

(ii) $f=\frac{\text { the function } \sin }{\text { the function } \exp \text {, }}$

(iii) $Z \subseteq \operatorname{dom}\left(\left(-\frac{1}{2}\right) \frac{\text { (the function sin) }+(\text { the function } \cos )}{\text { the function } \exp }\right)$,

(iv) $Z=\operatorname{dom} f$, and

(v) $f$ is continuous on $A$.

Then $\int_{A} f(x) d x=\left(\left(-\frac{1}{2}\right) \frac{(\text { the function } \sin )+(\text { the function } \cos )}{\text { the function } \exp }\right)(\sup A)-$ $\left(\left(-\frac{1}{2}\right) \frac{(\text { the function } \sin )+(\text { the function } \cos )}{\text { the function } \exp }\right)(\inf A)$.

(9) Suppose $Z \subseteq \operatorname{dom}\left(\frac{1}{2} \frac{\text { (the function sin)-(the function cos) }}{\text { the function } \exp }\right)$. Then

(i) $\frac{1}{2} \frac{\text { (the function sin)-(the function } \cos \text { ) }}{\text { the function } \exp }$ is differentiable on $Z$, and

(ii) for every $x$ such that $x \in Z$ holds

$\left(\frac{1}{2} \frac{\text { (the function sin) }-(\text { the function } \cos )}{\text { the function exp }}\right)^{\prime}{ }_{Z}(x)=\frac{\text { (the function } \cos )(x)}{\text { (the function } \exp )(x)}$.

(10) Suppose that

(i) $A \subseteq Z$,

(ii) $f=\frac{\text { the function } \cos }{\text { the function } \exp }$,

(iii) $Z \subseteq \operatorname{dom}\left(\frac{1}{2} \frac{\text { (the function sin)-(the function } \cos )}{\text { the function } \exp }\right)$,

(iv) $Z=\operatorname{dom} f$, and

(v) $f$ is continuous on $A$.

Then $\int_{A} f(x) d x=\left(\frac{1}{2} \frac{(\text { the function } \sin )-(\text { the function } \cos )}{\text { the function } \exp }\right)(\sup A)-$ $\left(\frac{1}{2} \frac{(\text { the function } \sin )-(\text { the function } \cos )}{\text { the function } \exp }\right)(\inf A)$.

(11) Suppose that

(i) $A \subseteq Z$,

(ii) $f=($ the function $\exp )(($ the function $\sin )+($ the function cos $))$,

(iii) $Z \subseteq \operatorname{dom}(($ the function $\exp )$ (the function $\sin )$ ),

(iv) $Z=\operatorname{dom} f$, and

(v) $f$ is continuous on $A$.

Then $\int_{A} f(x) d x=(($ the function $\exp ) \quad($ the function $\sin ))(\sup A)-(($ the function $\exp )($ the function $\sin ))(\inf A)$.

(12) Suppose that

(i) $A \subseteq Z$, 
(ii) $f=($ the function $\exp )(($ the function $\cos )-($ the function $\sin ))$,

(iii) $Z \subseteq \operatorname{dom}($ (the function $\exp$ ) (the function cos)),

(iv) $Z=\operatorname{dom} f$, and

(v) $f$ is continuous on $A$.

Then $\int_{A} f(x) d x=(($ the function $\exp )$ (the function $\left.\cos )\right)(\sup A)-(($ the function exp) (the function $\cos ))(\inf A)$.

(13) Suppose that

(i) $A \subseteq Z$,

(ii) $f_{1}=\square^{2}$,

(iii) $f=-\frac{\frac{\text { the function } \sin }{\text { the function } \cos }}{f_{1}}+\frac{\frac{1}{\text { id } Z}}{(\text { the function } \cos )^{2}}$,

(iv) $Z \subseteq \operatorname{dom}\left(\frac{1}{\operatorname{id} Z}\right.$ (the function tan)),

(v) $Z=\operatorname{dom} f$, and

(vi) $f$ is continuous on $A$.

Then $\int_{A} f(x) d x=\left(\frac{1}{\mathrm{id}_{Z}}\right.$ (the function $\left.\left.\tan \right)\right)(\sup A)-\left(\frac{1}{\mathrm{id} Z}\right.$ (the function $\tan ))(\inf A)$.

(14) Suppose that

(i) $A \subseteq Z$,

(ii) $f=-\frac{\frac{\text { the function } \cos }{\text { the function } \sin }}{f_{1}}-\frac{\frac{1}{\mathrm{id} Z}}{(\text { the function } \sin )^{2}}$,

(iii) $f_{1}=\square^{2}$,

(iv) $Z \subseteq \operatorname{dom}\left(\frac{1}{\operatorname{id}_{Z}}\right.$ (the function cot)),

(v) $Z=\operatorname{dom} f$, and

(vi) $f$ is continuous on $A$.

Then $\int_{A} f(x) d x=\left(\frac{1}{\mathrm{id}_{Z}}\right.$ (the function $\left.\left.\cot \right)\right)(\sup A)-\left(\frac{1}{\mathrm{id}_{Z}}\right.$ (the function $\cot ))(\inf A)$.

(15) Suppose that

(i) $A \subseteq Z$,

(ii) $f=\frac{\frac{\text { the function } \sin }{\text { the function cos }}}{\text { id } Z}+\frac{\text { the function } \ln }{\text { (the function } \cos )^{2}}$,

(iii) $Z \subseteq \operatorname{dom}(($ the function $\ln )$ (the function $\tan )$ ),

(iv) $Z=\operatorname{dom} f$, and

(v) $f$ is continuous on $A$.

Then $\int_{A} f(x) d x=(($ the function $\ln )$ (the function $\left.\tan )\right)(\sup A)-(($ the function $\ln )($ the function $\tan ))(\inf A)$.

(16) Suppose that

(i) $A \subseteq Z$,

(ii) $f=\frac{\frac{\text { the function } \cos }{\text { the function } \sin }}{\text { id } Z}-\frac{\text { the function } \ln }{\text { (the function } \sin )^{2}}$, 
(iii) $Z \subseteq \operatorname{dom}(($ the function $\ln )$ (the function cot)),

(iv) $Z=\operatorname{dom} f$, and

(v) $f$ is continuous on $A$.

Then $\int_{A} f(x) d x=(($ the function $\ln )$ (the function $\left.\cot )\right)(\sup A)-(($ the function $\ln )($ the function $\cot ))(\inf A)$.

(17) Suppose that

(i) $A \subseteq Z$,

(ii) $f=\frac{\text { the function } \tan }{\operatorname{id}_{Z}}+\frac{\text { the function } \ln }{\text { (the function } \cos )^{2}}$,

(iii) $Z \subseteq \operatorname{dom}(($ the function $\ln )$ (the function $\tan )$ ),

(iv) $Z \subseteq \operatorname{dom}$ (the function $\tan$ ),

(v) $Z=\operatorname{dom} f$, and

(vi) $f$ is continuous on $A$.

Then $\int_{A} f(x) d x=(($ the function $\ln ) \quad($ the function $\tan ))(\sup A)-(($ the function $\ln )($ the function $\tan ))(\inf A)$.

(18) Suppose that

(i) $A \subseteq Z$,

(ii) $f=\frac{\text { the function cot }}{\text { id }_{Z}}-\frac{\text { the function } \ln }{\text { (the function } \sin )^{2}}$,

(iii) $Z \subseteq \operatorname{dom}(($ the function $\ln )$ (the function cot)),

(iv) $Z \subseteq \operatorname{dom}$ (the function cot),

(v) $Z=\operatorname{dom} f$, and

(vi) $f$ is continuous on $A$.

Then $\int_{A} f(x) d x=(($ the function $\ln )$ (the function $\left.\cot )\right)(\sup A)-(($ the function $\ln )($ the function $\cot ))(\inf A)$.

(19) Suppose that

(i) $A \subseteq Z$,

(ii) for every $x$ such that $x \in Z$ holds $f_{1}(x)=1$,

(iii) $f=\frac{\text { the function } \arctan }{\text { id } Z}+\frac{\text { the function } \ln }{f_{1}+\square^{2}}$,

(iv) $Z \subseteq]-1,1[$,

(v) $Z=\operatorname{dom} f$, and

(vi) $f$ is continuous on $A$.

Then $\int_{A} f(x) d x=(($ the function $\ln )($ the function $\arctan ))(\sup A)-(($ the function ln) (the function $\arctan ))(\inf A)$.

(20) Suppose that

(i) $A \subseteq Z$,

(ii) for every $x$ such that $x \in Z$ holds $f_{1}(x)=1$,

(iii) $f=\frac{\text { the function arccot }}{\operatorname{id} z}-\frac{\text { the function } \ln }{f_{1}+\square^{2}}$, 
(iv) $Z \subseteq]-1,1[$,

(v) $Z=\operatorname{dom} f$, and

(vi) $\quad f$ is continuous on $A$.

Then $\int_{A} f(x) d x=(($ the function $\ln )$ (the function $\left.\operatorname{arccot})\right)(\sup A)-(($ the function $\ln )$ (the function $\operatorname{arccot}))(\inf A)$.

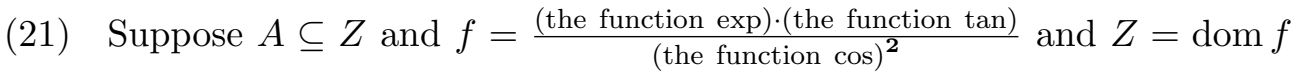
and $f$ is continuous on $A$. Then $\int_{A} f(x) d x=(($ the function exp) $\cdot$ (the function $\tan ))(\sup A)-(($ the function $\exp ) \cdot($ the function $\tan ))(\inf A)$.

(22) Suppose $A \subseteq Z$ and $f=-\frac{\text { (the function exp).(the function cot) }}{{\text { (the function } \sin )^{2}}}$ and $Z=\operatorname{dom} f$ and $f$ is continuous on $A$. Then $\int_{A} f(x) d x=(($ the function $\exp ) \cdot($ the function $\cot ))(\sup A)-(($ the function $\exp ) \cdot($ the function $\cot ))(\inf A)$.

(23) Suppose $Z \subseteq \operatorname{dom}(($ the function exp) $\cdot($ the function cot $))$. Then

(i) - (the function $\exp$ ) ( the function cot) is differentiable on $Z$, and

(ii) for every $x$ such that $x \in Z$ holds (-(the function exp) · (the function cot) $)^{\prime}{ }_{Z}(x)=\frac{\text { (the function exp) }((\text { the function cot })(x))}{\left(\text { the function sin) }(x)^{2}\right.}$.

(24) Suppose $A \subseteq Z$ and $f=\frac{\text { (the function exp).(the function cot) }}{\text { (the function sin) }^{2}}$ and $Z=\operatorname{dom} f$ and $f$ is continuous on $A$. Then $\int_{A} f(x) d x=(-($ the function exp) .

$($ the function $\cot ))(\sup A)-(-($ the function $\exp ) \cdot($ the function $\cot ))(\inf A)$.

(25) Suppose that

(i) $A \subseteq Z$,

(ii) $f=\frac{1}{\operatorname{id}_{Z}((\text { the function } \cos ) \cdot(\text { the function } \ln ))^{2}}$,

(iii) $Z \subseteq \operatorname{dom}(($ the function $\tan ) \cdot($ the function $\ln ))$,

(iv) $Z=\operatorname{dom} f$, and

(v) $f$ is continuous on $A$.

Then $\int_{A} f(x) d x=(($ the function $\tan ) \cdot($ the function $\ln ))(\sup A)-(($ the function $\tan ) \cdot($ the function $\ln ))(\inf A)$.

(26) Suppose that

(i) $A \subseteq Z$,

(ii) $f=-\frac{1}{\operatorname{id}_{Z}((\text { the function } \sin ) \cdot(\text { the function } \ln ))^{2}}$,

(iii) $Z \subseteq \operatorname{dom}(($ the function cot) $\cdot($ the function $\ln ))$,

(iv) $Z=\operatorname{dom} f$, and

(v) $f$ is continuous on $A$. 
Then $\int_{A} f(x) d x=(($ the function cot $) \cdot($ the function $\ln ))(\sup A)-(($ the function $\cot ) \cdot($ the function $\ln ))(\inf A)$.

(27) Suppose $Z \subseteq \operatorname{dom}(($ the function cot) $\cdot($ the function ln)). Then

(i) $\quad$ (the function cot) - (the function $\ln$ ) is differentiable on $Z$, and

(ii) for every $x$ such that $x \in Z$ holds (-(the function cot) $\cdot$ (the function $\ln ))_{\uparrow Z}^{\prime}(x)=\frac{1}{x \cdot\left(\text { the function sin) }((\text { the function } \ln )(x))^{2}\right.}$.

(28) Suppose that

(i) $A \subseteq Z$,

(ii) $f=\frac{1}{\operatorname{id}_{Z}((\text { the function } \sin ) \cdot(\text { the function } \ln ))^{2}}$,

(iii) $Z \subseteq \operatorname{dom}(($ the function cot) $\cdot($ the function $\ln ))$,

(iv) $Z=\operatorname{dom} f$, and

(v) $f$ is continuous on $A$.

Then $\int_{A} f(x) d x=(-($ the function $\cot ) \cdot($ the function $\ln ))(\sup A)-(-($ the function cot $) \cdot($ the function $\ln ))(\inf A)$.

(29) Suppose that

(i) $A \subseteq Z$,

(ii) $f=\frac{\text { the function exp }}{((\text { the function } \cos ) \cdot(\text { the function } \exp ))^{2}}$,

(iii) $Z \subseteq \operatorname{dom}(($ the function $\tan ) \cdot($ the function $\exp ))$,

(iv) $Z=\operatorname{dom} f$, and

(v) $f$ is continuous on $A$.

Then $\int_{A} f(x) d x=(($ the function $\tan ) \cdot($ the function $\exp ))(\sup A)-(($ the function $\tan ) \cdot($ the function $\exp ))(\inf A)$.

(30) Suppose that

(i) $A \subseteq Z$,

(ii) $f=-\frac{\text { the function } \exp }{((\text { the function sin).(the function } \exp ))^{2}}$,

(iii) $Z \subseteq \operatorname{dom}($ (the function cot) $\cdot($ the function $\exp ))$,

(iv) $Z=\operatorname{dom} f$, and

(v) $f$ is continuous on $A$.

Then $\int_{A} f(x) d x=(($ the function cot $) \cdot($ the function $\exp ))(\sup A)-(($ the function cot) $\cdot($ the function $\exp ))(\inf A)$.

(31) Suppose $Z \subseteq \operatorname{dom}(($ the function cot) $\cdot($ the function exp)). Then

(i) - (the function cot) - (the function exp) is differentiable on $Z$, and

(ii) for every $x$ such that $x \in Z$ holds (-(the function cot) $\cdot$ (the function $\exp ))^{\prime}{ }_{\uparrow}(x)=\frac{(\text { the function exp })(x)}{\left(\text { the function sin) }((\text { the function } \exp )(x))^{2}\right.}$.

(32) Suppose that

(i) $A \subseteq Z$, 
(ii) $\quad f=\frac{\text { the function } \exp }{\left((\text { the function sin) } \cdot(\text { the function } \exp ))^{2}\right.}$,

(iii) $Z \subseteq \operatorname{dom}(($ the function cot) $\cdot($ the function $\exp ))$,

(iv) $Z=\operatorname{dom} f$, and

(v) $f$ is continuous on $A$.

Then $\int_{A} f(x) d x=(-($ the function $\cot ) \cdot($ the function $\exp ))(\sup A)-(-($ the function cot $) \cdot($ the function $\exp ))(\inf A)$.

(33) Suppose that

(i) $A \subseteq Z$,

(ii) for every $x$ such that $x \in Z$ holds $f(x)=-\frac{1}{x^{2} \cdot(\text { the function } \cos )\left(\frac{1}{x}\right)^{2}}$,

(iii) $Z \subseteq \operatorname{dom}\left((\right.$ the function $\left.\tan ) \cdot \frac{1}{\operatorname{id} z}\right)$,

(iv) $Z=\operatorname{dom} f$, and

(v) $f$ is continuous on $A$.

Then $\int_{A} f(x) d x=\left((\right.$ the function $\left.\tan ) \cdot \frac{1}{\operatorname{id} Z}\right)(\sup A)-(($ the function $\tan )$ $\left.\cdot \frac{1}{\operatorname{id}_{Z}}\right)(\inf A)$.

(34) Suppose $Z \subseteq \operatorname{dom}\left((\right.$ the function $\left.\tan ) \cdot \frac{1}{\mathrm{id} Z}\right)$. Then

(i) - (the function $\tan ) \cdot \frac{1}{\mathrm{id}_{Z}}$ is differentiable on $Z$, and

(ii) for every $x$ such that $x \in Z$ holds $\left(-(\text { the function } \tan ) \cdot \frac{1}{\mathrm{id}_{Z}}\right)_{{ }^{\prime} Z}^{\prime}(x)=$ $\frac{1}{x^{2} \cdot(\text { the function } \cos )\left(\frac{1}{x}\right)^{2}}$.

(35) Suppose that

(i) $A \subseteq Z$,

(ii) for every $x$ such that $x \in Z$ holds $f(x)=\frac{1}{\left.x^{2} \text {.(the function } \cos \right)\left(\frac{1}{x}\right)^{2}}$,

(iii) $Z \subseteq \operatorname{dom}\left((\right.$ the function $\left.\tan ) \cdot \frac{1}{\operatorname{id} Z}\right)$,

(iv) $Z=\operatorname{dom} f$, and

(v) $f$ is continuous on $A$.

Then $\int_{A} f(x) d x=\left(-(\right.$ the function $\left.\tan ) \cdot \frac{1}{\mathrm{id}_{Z}}\right)(\sup A)-(-($ the function $\left.\tan ) \cdot \frac{1}{\operatorname{id} z}\right)(\inf A)$.

(36) Suppose that

(i) $A \subseteq Z$,

(ii) for every $x$ such that $x \in Z$ holds $f(x)=\frac{1}{x^{2} \cdot(\text { the function } \sin )\left(\frac{1}{x}\right)^{2}}$,

(iii) $Z \subseteq \operatorname{dom}\left((\right.$ the function $\left.\cot ) \cdot \frac{1}{\operatorname{id}_{Z}}\right)$,

(iv) $Z=\operatorname{dom} f$, and

(v) $f$ is continuous on $A$.

Then $\int_{A} f(x) d x=\left((\right.$ the function $\left.\cot ) \cdot \frac{1}{\mathrm{id}_{Z}}\right)(\sup A)-(($ the function $\cot )$ $\left.\cdot \frac{1}{\operatorname{id}_{Z}}\right)(\inf A)$. 
(37) Suppose that $A \subseteq Z$ and for every $x$ such that $x \in Z$ holds $f_{1}(x)=1$ and (the function $\arctan )(x)>0$ and $f=\frac{1}{\left(f_{1}+\square^{2}\right) \text { the function } \arctan }$ and $Z \subseteq$ ]-1, $1[$ and $Z \subseteq \operatorname{dom}(($ the function $\ln ) \cdot($ the function $\arctan ))$ and $Z=\operatorname{dom} f$ and $f$ is continuous on $A$. Then $\int_{A} f(x) d x=(($ the function $\ln ) \cdot($ the function $\arctan ))(\sup A)-(($ the function $\ln ) \cdot($ the function $\arctan ))(\inf A)$.

(38) Suppose that $A \subseteq Z$ and $f=n \frac{\left(\square^{n-1}\right) \cdot \text { the function arctan }}{f_{1}+\square^{2}}$ and for every $x$ such that $x \in Z$ holds $f_{1}(x)=1$ and $\left.Z \subseteq\right]-1,1\left[\right.$ and $Z \subseteq \operatorname{dom}\left(\left(\square^{n}\right) \cdot\right.$ the function $\arctan )$ and $Z=\operatorname{dom} f$ and $f$ is continuous on $A$. Then $\int_{A} f(x) d x=\left(\left(\square^{n}\right) \cdot\right.$ the function $\arctan )(\sup A)-\left(\left(\square^{n}\right) \cdot\right.$ the function $\left.\arctan \right)(\inf A)$.

(39) Suppose that $A \subseteq Z$ and for every $x$ such that $x \in Z$ holds $f_{1}(x)=1$ and $f=-n \frac{\left(\square^{n-1}\right) \cdot \text { the function arccot }}{f_{1}+\square^{2}}$ and $\left.Z \subseteq\right]-1,1\left[\right.$ and $Z \subseteq \operatorname{dom}\left(\left(\square^{n}\right) \cdot\right.$ the function arccot) and $Z=\operatorname{dom} f$ and $f$ is continuous on $A$. Then $\int_{A} f(x) d x=\left(\left(\square^{n}\right) \cdot\right.$ the function $\operatorname{arccot})(\sup A)-\left(\left(\square^{n}\right) \cdot\right.$ the function $\left.\operatorname{arccot}\right)(\inf A)$.

(40) Suppose $Z \subseteq \operatorname{dom}\left(\left(\square^{n}\right) \cdot\right.$ the function arccot $)$ and $\left.Z \subseteq\right]-1,1[$. Then

(i) $\quad-\left(\square^{n}\right) \cdot$ the function arccot is differentiable on $Z$, and

(ii) for every $x$ such that $x \in Z$ holds $\left(-\left(\square^{n}\right) \cdot \text { the function } \operatorname{arccot}\right)_{\mid Z}^{\prime}(x)=$ $\frac{n \cdot(\text { the function } \operatorname{arccot})(x)^{n-1}}{1+x^{2}}$.

(41) Suppose that $A \subseteq Z$ and for every $x$ such that $x \in Z$ holds $f_{1}(x)=1$ and $f=n \frac{\left(\square^{n-1}\right) \cdot \text { the function arccot }}{f_{1}+\square^{2}}$ and $\left.Z \subseteq\right]-1,1\left[\right.$ and $Z \subseteq \operatorname{dom}\left(\left(\square^{n}\right) \cdot\right.$ the function arccot) and $Z=\operatorname{dom} f$ and $f$ is continuous on $A$. Then $\int_{A} f(x) d x=$ $\left(-\left(\square^{n}\right) \cdot\right.$ the function $\left.\operatorname{arccot}\right)(\sup A)-\left(-\left(\square^{n}\right) \cdot\right.$ the function $\left.\operatorname{arccot}\right)(\inf A)$.

(42) Suppose that $A \subseteq Z$ and for every $x$ such that $x \in Z$ holds $f_{1}(x)=1$ and $f=\frac{\text { the function arctan }}{f_{1}+\square^{2}}$ and $\left.Z \subseteq\right]-1,1\left[\right.$ and $Z \subseteq \operatorname{dom}\left(\left(\square^{2}\right) \cdot\right.$ the function arctan $)$ and $Z=\operatorname{dom} f$ and $f$ is continuous on $A$. Then $\int_{A} f(x) d x=\left(\frac{1}{2}\left(\left(\square^{2}\right) \cdot\right.\right.$ the function $\arctan ))(\sup A)-\left(\frac{1}{2}\left(\left(\square^{2}\right) \cdot\right.\right.$ the function $\left.\left.\arctan \right)\right)(\inf A)$.

(43) Suppose that $A \subseteq Z$ and for every $x$ such that $x \in Z$ holds $f_{1}(x)=1$ and $f=-\frac{\text { the function arccot }}{f_{1}+\square^{2}}$ and $\left.Z \subseteq\right]-1,1\left[\right.$ and $Z \subseteq \operatorname{dom}\left(\left(\square^{2}\right) \cdot\right.$ the function arccot) and $Z=\operatorname{dom} f$ and $f$ is continuous on $A$. Then $\int_{A} f(x) d x=\left(\frac{1}{2}\left(\left(\square^{2}\right) \cdot\right.\right.$ the function $\operatorname{arccot}))(\sup A)-\left(\frac{1}{2}\left(\left(\square^{2}\right) \cdot\right.\right.$ the function $\left.\left.\operatorname{arccot}\right)\right)(\inf A)$.

(44) Suppose $Z \subseteq \operatorname{dom}\left(\left(\square^{2}\right) \cdot\right.$ the function arccot) and $\left.Z \subseteq\right]-1,1[$. Then

(i) $\quad-\frac{1}{2}\left(\left(\square^{2}\right) \cdot\right.$ the function arccot) is differentiable on $Z$, and

(ii) for every $x$ such that $x \in Z$ holds 
$\left(-\frac{1}{2}\left(\left(\square^{2}\right) \cdot \text { the function } \operatorname{arccot}\right)\right)_{\lceil Z}^{\prime}(x)=\frac{\text { (the function } \operatorname{arccot})(x)}{1+x^{2}}$.

(45) Suppose that $A \subseteq Z$ and for every $x$ such that $x \in Z$ holds $f_{1}(x)=1$ and $f=\frac{\text { the function arccot }}{f_{1}+\square^{2}}$ and $\left.Z \subseteq\right]-1,1[$ and $Z \subseteq$ $\operatorname{dom}\left(\left(\square^{2}\right)\right.$. the function arccot) and $Z=\operatorname{dom} f$ and $f$ is continuous on $A$. Then $\int_{A} f(x) d x=\left(-\frac{1}{2}\left(\left(\square^{2}\right) \cdot\right.\right.$ the function $\left.\left.\operatorname{arccot}\right)\right)(\sup A)-$ $\left(-\frac{1}{2}\left(\left(\square^{2}\right) \cdot\right.\right.$ the function $\left.\left.\operatorname{arccot}\right)\right)(\inf A)$.

(46) Suppose that

(i) $A \subseteq Z$,

(ii) for every $x$ such that $x \in Z$ holds $f_{1}(x)=1$,

(iii) $f=($ the function $\arctan )+\frac{\operatorname{id}_{Z}}{f_{1}+\square^{2}}$,

(iv) $Z \subseteq]-1,1[$,

(v) $Z=\operatorname{dom} f$, and

(vi) $f$ is continuous on $A$.

Then $\int_{A} f(x) d x=\left(\operatorname{id}_{Z}\right.$ the function $\left.\arctan \right)(\sup A)-\left(\operatorname{id}_{Z}\right.$ the function $\arctan )(\inf A)$.

(47) Suppose that

(i) $A \subseteq Z$,

(ii) for every $x$ such that $x \in Z$ holds $f_{1}(x)=1$,

(iii) $f=($ the function $\operatorname{arccot})-\frac{\operatorname{id} Z}{f_{1}+\square^{2}}$,

(iv) $Z \subseteq]-1,1[$,

(v) $Z=\operatorname{dom} f$, and

(vi) $f$ is continuous on $A$.

Then $\int_{A} f(x) d x=\left(\operatorname{id}_{Z}\right.$ the function $\left.\operatorname{arccot}\right)(\sup A)-\left(\operatorname{id}_{Z}\right.$ the function $\operatorname{arccot})(\inf A)$.

(48) Suppose that

(i) $A \subseteq Z$,

(ii) $Z \subseteq]-1,1[$,

(iii) $f=\frac{\text { (the function exp).(the function arctan) }}{f_{1}+\square^{2}}$,

(iv) for every $x$ such that $x \in Z$ holds $f_{1}(x)=1$,

(v) $Z=\operatorname{dom} f$, and

(vi) $f$ is continuous on $A$.

Then $\int_{A} f(x) d x=(($ the function $\exp ) \cdot($ the function $\arctan ))(\sup A)-(($ the function $\exp ) \cdot($ the function $\arctan ))(\inf A)$.

(49) Suppose that

(i) $A \subseteq Z$,

(ii) $Z \subseteq]-1,1[$, 
(iii) $f=-\frac{\text { (the function } \exp ) \cdot(\text { the function arccot) }}{f_{1}+\square^{2}}$,

(iv) for every $x$ such that $x \in Z$ holds $f_{1}(x)=1$,

(v) $Z=\operatorname{dom} f$, and

(vi) $f$ is continuous on $A$.

Then $\int_{A} f(x) d x=(($ the function $\exp ) \cdot($ the function $\operatorname{arccot}))(\sup A)-(($ the function $\exp ) \cdot($ the function $\operatorname{arccot}))(\inf A)$.

(50) Suppose $Z \subseteq \operatorname{dom}(($ the function $\exp ) \cdot($ the function arccot)) and $Z \subseteq]-1,1[$. Then

(i) $\quad$ (the function exp) - (the function arccot) is differentiable on $Z$, and

(ii) for every $x$ such that $x \in Z$ holds (-(the function exp) $\cdot$ (the function $\operatorname{arccot}))_{{ }^{\prime}}^{\prime}(x)=\frac{\text { (the function exp })((\text { the function } \operatorname{arccot})(x))}{1+x^{2}}$.

(51) Suppose that

(i) $A \subseteq Z$,

(ii) $Z \subseteq]-1,1[$,

(iii) $f=\frac{\text { (the function } \exp \text { ).(the function arccot) }}{f_{1}+\square^{2}}$,

(iv) for every $x$ such that $x \in Z$ holds $f_{1}(x)=1$,

(v) $Z=\operatorname{dom} f$, and

(vi) $\quad f$ is continuous on $A$.

Then $\int_{A} f(x) d x=(-($ the function $\exp ) \cdot($ the function $\operatorname{arccot}))(\sup A)-$ $(-($ the function $\exp ) \cdot($ the function $\operatorname{arccot}))(\inf A)$.

(52) Suppose that $A \subseteq Z \subseteq \operatorname{dom}\left((\right.$ the function $\left.\ln ) \cdot\left(f_{1}+f_{2}\right)\right)$ and $f=\frac{\operatorname{id} Z}{f_{1}+f_{2}}$ and $f_{2}=\square^{2}$ and for every $x$ such that $x \in Z$ holds $f_{1}(x)=1$ and $Z=\operatorname{dom} f$ and $f$ is continuous on $A$. Then $\int_{A} f(x) d x=\left(\frac{1}{2}\left((\right.\right.$ the function $\left.\left.\ln ) \cdot\left(f_{1}+f_{2}\right)\right)\right)(\sup A)-$ $\left(\frac{1}{2}\left((\right.\right.$ the function $\left.\left.\ln ) \cdot\left(f_{1}+f_{2}\right)\right)\right)(\inf A)$.

(53) Suppose that $A \subseteq Z \subseteq \operatorname{dom}\left((\right.$ the function $\left.\ln ) \cdot\left(f_{1}+f_{2}\right)\right)$ and $f=\frac{\operatorname{id}_{Z}}{a\left(f_{1}+f_{2}\right)}$ and for every $x$ such that $x \in Z$ holds $h(x)=\frac{x}{a}$ and $f_{1}(x)=1$ and $a \neq 0$ and $f_{2}=$ $\left(\square^{2}\right) \cdot h$ and $Z=\operatorname{dom} f$ and $f$ is continuous on $A$. Then $\int_{A} f(x) d x=\left(\frac{a}{2}\right.$ ((the function $\left.\left.\ln ) \cdot\left(f_{1}+f_{2}\right)\right)\right)(\sup A)-\left(\frac{a}{2}\left((\right.\right.$ the function $\left.\left.\ln ) \cdot\left(f_{1}+f_{2}\right)\right)\right)(\inf A)$.

(54) Suppose $Z \subseteq \operatorname{dom}\left(\frac{1}{\operatorname{id}_{Z}}\right.$ the function arctan) and $\left.Z \subseteq\right]-1,1[$. Then

(i) $-\frac{1}{\mathrm{id} Z}$ the function $\arctan$ is differentiable on $Z$, and

(ii) for every $x$ such that $x \in Z$ holds $\left(-\frac{1}{\mathrm{id}_{Z}} \text { the function } \arctan \right)^{\prime}{ }_{{ }^{\prime}}(x)=$ $\frac{(\text { the function } \arctan )(x)}{x^{2}}-\frac{1}{x \cdot\left(1+x^{2}\right)}$.

(55) Suppose $Z \subseteq \operatorname{dom}\left(\frac{1}{\operatorname{id}_{Z}}\right.$ the function arccot $)$ and $\left.Z \subseteq\right]-1,1[$. Then

(i) $-\frac{1}{\mathrm{id}_{Z}}$ the function arccot is differentiable on $Z$, and 
(ii) for every $x$ such that $x \in Z$ holds $\left(-\frac{1}{\mathrm{id}_{Z}} \text { the function } \operatorname{arccot}\right)_{{ }^{\prime} Z}^{\prime}(x)=$ $\frac{(\text { the function } \operatorname{arccot})(x)}{x^{2}}+\frac{1}{x \cdot\left(1+x^{2}\right)}$.

(56) Suppose that $A \subseteq Z$ and for every $x$ such that $x \in Z$ holds $f_{1}(x)=1$ and $f=\frac{\text { the function } \arctan }{\square^{2}}-\frac{1}{\operatorname{id}_{Z}\left(f_{1}+\square^{2}\right)}$ and $Z \subseteq \operatorname{dom}\left(\frac{1}{\operatorname{id}_{Z}}\right.$ the function arctan $)$ and $Z \subseteq]-1,1\left[\right.$ and $Z=\operatorname{dom} f$ and $f$ is continuous on $A$. Then $\int_{A} f(x) d x=$ $\left(-\frac{1}{\mathrm{id}_{Z}}\right.$ the function $\left.\arctan \right)(\sup A)-\left(-\frac{1}{\mathrm{id}_{Z}}\right.$ the function $\left.\arctan \right)(\inf A)$.

(57) Suppose that $A \subseteq Z$ and for every $x$ such that $x \in Z$ holds $f_{1}(x)=1$ and $f=\frac{\text { the function } \operatorname{arccot}}{\square^{2}}+\frac{1}{\operatorname{id}_{Z}\left(f_{1}+\square^{2}\right)}$ and $Z \subseteq \operatorname{dom}\left(\frac{1}{\operatorname{id}_{Z}}\right.$ the function arccot) and $Z \subseteq]-1,1\left[\right.$ and $Z=\operatorname{dom} f$ and $f$ is continuous on $A$. Then $\int_{A} f(x) d x=$ $\left(-\frac{1}{\operatorname{id}_{Z}}\right.$ the function $\left.\operatorname{arccot}\right)(\sup A)-\left(-\frac{1}{\operatorname{id}_{Z}}\right.$ the function $\left.\operatorname{arccot}\right)(\inf A)$.

\section{REFERENCES}

[1] Czesław Byliński. Partial functions. Formalized Mathematics, 1(2):357-367, 1990.

[2] Noboru Endou and Artur Korniłowicz. The definition of the Riemann definite integral and some related lemmas. Formalized Mathematics, 8(1):93-102, 1999.

[3] Noboru Endou, Katsumi Wasaki, and Yasunari Shidama. Definition of integrability for partial functions from $\mathbb{R}$ to $\mathbb{R}$ and integrability for continuous functions. Formalized Mathematics, 9(2):281-284, 2001.

[4] Krzysztof Hryniewiecki. Basic properties of real numbers. Formalized Mathematics, 1(1):35-40, 1990.

[5] Jarosław Kotowicz. Convergent real sequences. Upper and lower bound of sets of real numbers. Formalized Mathematics, 1(3):477-481, 1990.

[6] Jarosław Kotowicz. Partial functions from a domain to a domain. Formalized Mathematics, 1(4):697-702, 1990.

[7] Jarosław Kotowicz. Partial functions from a domain to the set of real numbers. Formalized Mathematics, 1(4):703-709, 1990.

[8] Jarosław Kotowicz. Real sequences and basic operations on them. Formalized Mathematics, 1(2):269-272, 1990.

[9] Jarosław Kotowicz. The limit of a real function at infinity. Formalized Mathematics, 2(1):17-28, 1991.

[10] Xiquan Liang and Bing Xie. Inverse trigonometric functions arctan and arccot. Formalized Mathematics, 16(2):147-158, 2008, doi:10.2478/v10037-008-0021-3.

[11] Konrad Raczkowski. Integer and rational exponents. Formalized Mathematics, 2(1):125130, 1991.

[12] Konrad Raczkowski and Paweł Sadowski. Real function continuity. Formalized Mathematics, 1(4):787-791, 1990.

[13] Konrad Raczkowski and Paweł Sadowski. Real function differentiability. Formalized Mathematics, 1(4):797-801, 1990.

[14] Konrad Raczkowski and Paweł Sadowski. Topological properties of subsets in real numbers. Formalized Mathematics, 1(4):777-780, 1990.

[15] Yasunari Shidama. The Taylor expansions. Formalized Mathematics, 12(2):195-200, 2004.

[16] Andrzej Trybulec and Czesław Byliński. Some properties of real numbers. Formalized Mathematics, 1(3):445-449, 1990.

[17] Zinaida Trybulec. Properties of subsets. Formalized Mathematics, 1(1):67-71, 1990.

[18] Edmund Woronowicz. Relations defined on sets. Formalized Mathematics, 1(1):181-186, 1990. 
[19] Yuguang Yang and Yasunari Shidama. Trigonometric functions and existence of circle ratio. Formalized Mathematics, 7(2):255-263, 1998.

Received February 4, 2010 